\title{
18. Insuffisance rénale / Dialyse
}

\section{Congrès JASFGG 2014, Paris, France}

(C) Lavoisier SAS 2014

\section{P6.18-37}

\section{Entre Takotsubo et rhabdomyolyse, mon coeur balance}

\section{F. Weill ${ }^{1, *}$, C. Jehl ${ }^{1}$, A. Marti ${ }^{1}$, G. Kaltenbach ${ }^{1}$, T. Vogel ${ }^{1}$}

${ }^{1}$ Pôle de Gériatrie, Hôpitaux Universitaires de Strasbourg, Strasbourg, France

Introduction : Une rhabdomyolyse, le plus souvent sans conséquence, est fréquemment observée après une chute avec station prolongée au sol chez les personnes âgées. Les rhabdomyolyses massives $(\mathrm{CPK}>10$ $000 \mathrm{UI} / \mathrm{ml}$ ) peuvent cependant se compliquer d'insuffisance rénale aiguë. Par ailleurs, une élévation modérée de la troponine est possible dans les rhabdomyolyses sans que cette élévation ait une étiologie cardiaque.

Objectifs : Discuter autour d'un cas l'association entre élévation des CPK et de la tropinine.

Méthodes : Description d'un cas de rhabdomyolyse massive associée à une élévation importante de la troponine.

Résultats : la patiente âgée de 88 ans a chuté avec une station au sol estimée à 20 heures. Au SAU, le bilan biologique retrouvait des CPK à $63000 \mathrm{UI} / 1$, une troponine I à $17 \mu \mathrm{g} / 1$ et une insuffisance rénale aiguë avec une créatinine à $300 \mu \mathrm{mol} / 1$ et une urée à $30 \mathrm{mmol} / \mathrm{l}$ (DFG à $13 \mathrm{ml} /$ min). L'ECG d'admission mettait en évidence un sus-décalage du seg- ment ST en V3, V4 et V5. L'échographie cardiaque retrouvait une akinésie apicale compatible avec un SCA ou un syndrome de Takotsubo. Un traitement par Aspégic et clopidogrel est instauré.

Sur le plan rénal, l'évolution est favorable après une hyperhydratation IV par $\mathrm{NaCl} 0.9 \%$ et $\mathrm{HCO} 3-1.4 \%$. La patiente n'aura pas eu besoin de dialyse, et la créatinine retournera à ses valeurs antérieures.

Sur le plan cardiaque, l'IRM réalisée 15 jours après montrait une disparition complète de l'akinésie apicale.

Conclusion : Cette présentation soulève deux problématiques :

- le diagnostic différentiel entre un syndrome coronarien aigu et un syndrome de Takotsubo. Ce dernier se caractérise par l'absence de séquelles d'ischémie cardiaque lors de l'évaluation à distance en l'absence de coronarographie ;

- une élévation non spécifique de la troponine peut être observée lors de rhabdomyolyse, de sepsis, ou d'intoxication à l'héroïne ou à la cocaïne. Cette augmentation est cependant modérée dans ces cas en raison de la spécificité cardiaque de la troponine I.

Dans cette observation, la très importante élévation de la troponine I s'explique plutôt par le syndrome de Takotsubo et non par la rhabdomyolyse seule, malgré l'insuffisance rénale aiguë associée.

Mots clés : Rhabdomyolyse - Syndrome coronarien aigu - Syndrome de Takotsubo - Insuffisance rénale

Congrès JASFGG 2014, Paris, France $(\bowtie)$

e-mail : info@jasfgg2014.com 